\title{
Risk factors involved in transmission of Toxoplasma gondii and Neospora caninum infection in rabbit farms in Northern Italy
}

\author{
Tereza Machacova', Eva Bartova', Kamil Sedlak², Marie Budikova³, Alessandra Piccirillo4 \\ ${ }^{1}$ Department of Biology and Wildlife Diseases, Faculty of Veterinary Hygiene and Ecology, University of Veterinary and \\ Pharmaceutical Sciences, Brno, Czech Republic \\ 2 Department of Virology and Serology, State Veterinary Institute Prague, Czech Republic \\ ${ }^{3}$ Department of Mathematics and Statistics, Faculty of Science, Masaryk University, Brno, Czech Republic \\ ${ }^{4}$ Department of Comparative Biomedicine and Food Science, University of Padua, Legnaro (Padua), Italy
}

Machacova T, Bartova E, Sedlak K, Budikova M, Piccirillo A. Risk factors involved in transmission of Toxoplasma gondii and Neospora caninum infection in rabbit farms in Northern Italy. Ann Agric Environ Med. 2015; 22(4): 677-679. doi: 10.5604/12321966.1185774

\begin{abstract}
I Abstract
Introduction and objective. In Italy, rabbits are frequently reared for meat production. The aim of the study was to find the seroprevalence of T. gondii and N. caninum parasites, and risk factors of infection in rabbit farms.

Material and methods. Blood samples from 260 apparently healthy breeding rabbits were collected on 13 commercial farms in Northern Italy. Rabbits were divided into categories according to age, number of births, breed, province and size of farm. Samples were tested for antibodies to T. gondii and N. caninum using the indirect fluorescence antibody test (IFAT); samples with a titre $\geq 50$ were considered positive.

Results. Antibodies to T. gondii and N. caninum were found in $38(14.6 \%)$ and $3(1.2 \%)$ rabbits, respectively. A statistically significant difference ( $p$-value $\leq 0.05$ ) was found only in T. gondii prevalence among different rabbit breeds and provinces. Conclusion. Rabbits from Northern Italy are at risk of T. gondii and N. caninum infection; however, it is lower compared to seroprevalence noted in other animal species or in humans.
\end{abstract}

I Key words

Toxoplasmosis, neosporosis, rabbits, serological survey, risk factors

\section{INTRODUCTION AND OBJECTIVES}

Toxoplasmosis is a disease that in sensitive hosts causes different clinical symptoms, affects their reproduction and can lead to death [1]. In livestock, it is also connected with economic losses because sheep, goats and rabbits belong to the animal species very susceptible to Toxoplasma gondii infection. In Europe, there are many serological studies focusing on detection of $T$. gondii antibodies in rabbits; however, to our knowledge there is no similar study from Italy. The only study from Italy was undertaken by Zanet et al. [2] who isolated the DNA of T. gondii from tissues of $2.1 \%$ of 144 wild rabbits (Sylvilagus floridanus).

The consumption of raw or undercooked meat originated from meat-producing animals, including rabbits, is a very important source of T. gondii infection in humans. Since toxoplasmosis is a zoonosis [3], and rabbits are frequently used for meat consumption in Italy, it is important to know the risk factors of $T$. gondii infection from consumption of rabbit meat for humans, and the important factors for the transmission of $T$. gondii among rabbits. The rabbits could be infected with T. gondii by the ingestion of food or water contaminated with T. gondii oocysts from cat faeces [3], or by transmission of T. gondii to offspring through transplacental infection [4]. Rabbits are usually without clinical symptoms, therefore detection of antibodies is very important in epizootiological

Address for correspondence: Eva Bártová, Department of Biology and Wildlife Diseases, Faculty of Veterinary Hygiene and Ecology, University of Veterinary and Pharmaceutical Sciences, Palackého tř. 1/3, 61242 Brno, Czech Republic

E-mail: bartovae@vfu.cz

Received: 06 November 2014; accepted: 13 February 2015 studies. Rabbits could be infected with T. gondii and simultaneously with similar parasite N. caninum. This is why the aim of the presented study was to detect both T. gondii and $N$. caninum antibodies in rabbits, and evaluate the risk factors of infection on rabbit farms.

\section{MATERIALS AND METHODS}

During 2009, blood samples were collected from the auricular vein of 260 apparently healthy breeding rabbits on 13 commercial rabbit farms in Northern Italy (Veneto Region). All farms were industrial-cycle type with breeding and growing units located in the same house, with the number of breeding and growing does ranging between $300-3,000$ and between 2,000-14,000, respectively. Breeding does were individually housed in wire cages equipped with a nest box. Rabbits were fed ad libitum and drinking water was administered through nipple watering systems. Artificial insemination by using external semen was practiced on all farms.

All sampled doe rabbit showed no signs of clinical symptoms. Data, including age, breed, number of births and health conditions, were obtained through questionnaires at the farms. The rabbits were divided into five age categories: $2-5$ months $(\mathrm{n}=59) ; \geq 5-7$ months $(\mathrm{n}=66) ; \geq 7-13$ months $(\mathrm{n}=74) ; \geq 13-30$ months $(\mathrm{n}=26)$; and rabbits with unknown age $(n=35)$; five categories according to number of births: 0 birth $(\mathrm{n}=60) ; 1-2$ births $(\mathrm{n}=71) ; 3-7$ births $(\mathrm{n}=57) ; 8-15$ births $(n=37)$; and rabbits with an unknown number of births $(n=35)$. Four commercial hybrid lines: $W(n=40)$, 
$X(n=160), Y(n=40)$, and $Z$ (crossbred of New Zealand White $\mathrm{x}$ Californian, $\mathrm{n}=20$ ), marked similarly as in the study of Lonardi et al. [5], were included in the current study. Doe rabbits were also divided according to the provinces in which they were bred: Padova $(n=20)$, Rovigo $(n=20)$, Treviso $(n=160)$ and Verona $(n=60)$; and the size of the farms: small farms $(<1,000$ does, $n=120)$ and large farms $(\geq 1,000$ does, $\mathrm{n}=140)$. Data are summarized in Table 1.

Each blood sample was centrifuged and removed serum was frozen at $-20^{\circ} \mathrm{C}$ until testing. Antibodies to T. gondii and $N$. caninum were detected by indirect fluorescence antibody test (IFAT) using a commercially available T. gondii antigen and N. caninum antigen (VMRD, Pullman, USA), respectively, and anti-rabbit IgG FITC conjugate (Sigma Aldrich, Czech Republic). Positive and negative control sera from an experimental study of domestic rabbits with $T$. gondii oocysts [6] were included in the tests. Sera were diluted with PBS two-fold, starting at 1:50. In the present study, cut-off titre 50 was used for both parasites, which is higher than in experimental studies (cut-off titre 20), to minimize the risk of false positive samples due to cross-reactivity.

Table 1. Characteristics of rabbits and results of Toxoplasma gondii and Neospora caninum seroprevalence (IFAT)

\begin{tabular}{|c|c|c|c|}
\hline Characteristics & $\begin{array}{l}\text { No. of rabbits } \\
\text { tested }\end{array}$ & $\begin{array}{c}\text { T.gondii } \\
\text { Positive (\%) }\end{array}$ & $\begin{array}{l}\text { N. caninum } \\
\text { Positive (\%) }\end{array}$ \\
\hline \multicolumn{4}{|l|}{ Gender } \\
\hline Female (does) & 260 & $38(14.6 \%)$ & $3(1.2 \%)$ \\
\hline \multicolumn{4}{|l|}{ Age categories (months) } \\
\hline $2-5$ & 59 & $4(6.8 \%)$ & $0(0 \%)$ \\
\hline$\geq 5-7$ & 66 & $8(12.1 \%)$ & $2(3 \%)$ \\
\hline$\geq 7-13$ & 74 & $10(13.5 \%)$ & $0(0 \%)$ \\
\hline$\geq 13-30$ & 26 & $3(11.5 \%)$ & $1(3.8 \%)$ \\
\hline not known & 35 & $13(37 \%)$ & $0(0 \%)$ \\
\hline \multicolumn{4}{|l|}{ No of births } \\
\hline 0 & 60 & $4(6.7 \%)$ & $0(0 \%)$ \\
\hline $1-2$ & 71 & $6(8.5 \%)$ & $2(2.8 \%)$ \\
\hline $3-7$ & 57 & $9(15.8 \%)$ & $0(0 \%)$ \\
\hline $8-15$ & 37 & $6(16.2 \%)$ & $1(2.7 \%)$ \\
\hline many but not known & 35 & $13(37 \%)$ & $0(0 \%)$ \\
\hline \multicolumn{4}{|l|}{ Breed } \\
\hline W & 40 & $3(7.5 \%)$ & $0(0 \%)$ \\
\hline$x$ & 160 & $23(14.4 \%)$ & $3(1.9 \%)$ \\
\hline $\mathrm{Y}$ & 40 & $12(30 \%)$ & $0(0 \%)$ \\
\hline Z & 20 & $0(0 \%)$ & $0(0 \%)$ \\
\hline \multicolumn{4}{|l|}{ Provincie } \\
\hline Padova & 20 & $0(0 \%)$ & $0(0 \%)$ \\
\hline Rovigo & 20 & $3(15 \%)$ & $0(0 \%)$ \\
\hline Treviso & 160 & $11(6.9 \%)$ & $3(1.9 \%)$ \\
\hline Verona & 60 & $24(40 \%)$ & $0(0 \%)$ \\
\hline \multicolumn{4}{|l|}{ Size of farm } \\
\hline Small farms ( $<1000$ does $)$ & 120 & $19(15.8 \%)$ & $1(0.8 \%)$ \\
\hline Large farms ( $\geq 1000$ does) & 140 & $19(13.6 \%)$ & $2(1.4 \%)$ \\
\hline \multicolumn{4}{|c|}{$\begin{array}{l}\text { In case of } T \text {. gondii, the results of chi-square test proved (with the risk of error not more thar } \\
5 \% \text { ) dependence on breed }(\mathrm{X} 2=12.64, \mathrm{df}=3, \mathrm{p} \text {-value }=0.005 \text { ) and on provinces }(\mathrm{X} 2=42.08, \mathrm{df}=3 \\
\mathrm{p} \text {-value }<0.05 \text { ). Multiple comparison method based on arcsine transformation proved that there } \\
\text { is difference in prevalence between breeds } \mathrm{X} \text { and } \mathrm{Z} \text { and between } \mathrm{Y} \text { and } \mathrm{Z} \text {; and also between } \\
\text { two provinces Padova and Rovigo, two provinces Padova and Verona and two province } \\
\text { Treviso and Verona. In case of } N \text {. caninum, statistical analysis could not be done because } \\
\text { small number of positive samples. }\end{array}$} \\
\hline
\end{tabular}

Seroprevalence was statistically analysed, considering the variables of age, number of births, breeds, province and size of farms. Data analysis was performed by ChiSquare test for independence resp. Fisher exact test for Tables $2 \times 2$ using STATISTICA Cz 12 [7]. Dependence on age and seroprevalence was evaluated by Wilcoxon test. Null hypothesis was tested to ascertain if $T$. gondii seroprevalence depended on age, number of births, breeds, province and size of farms. Multiple comparison method based on arcsine transformation was used to compare T. gondii prevalence between breeds and provinces. The differences were considered statistically significant when $p$-value was $\leq 0.05$.

\section{RESULTS AND DISCUSSION}

In the presented study, antibodies to T. gondii and N. caninum were found in 38 (14.6\%) and $3(1.2 \%)$ doe breeding rabbits, respectively. No co-infection between $T$. gondii and $N$. caninum was found. Titres of T. gondii antibodies were at a low level (50-100). To the best of the authors' knowledge, this is the first serological study focused on T. gondii and $N$. caninum seroprevalence in rabbits from Northern Italy, and the first detection of $N$. caninum antibodies in domestic rabbits in Europe. It could be concluded that the does from Northern Italy are at risk of $T$. gondii and N. caninum infection; however the seroprevalence is lower compared to the seroprevalences noted in other animal species, or in humans.

Similar T. gondii prevalence was obtained by the different methods used. Antibodies to T. gondii were detected, e.g. by indirect haemagglutination test in $23.4 \%(18 / 77)$ rabbits from China [8], by Enzyme-Linked Immuno Sorbent Assay (ELISA) in $15.5 \%(103 / 1883)$ rabbits from the Czech Republic [9] and in $11 \%(22 / 194)$ rabbits from Egypt [10], or by Modified Agglutination Test (MAT) in $16.3 \%$ (70/429) rabbits from Mexico [11].

The results of the present study, based on different risk factors (age, number of births, breeds, provinces and size of farm), are summarized in Table 1. Lower T. gondii and $N$. caninum prevalence $(6.8 \%$ and $0 \%$, respectively) were found in rabbits until 5 months of age, while in older rabbits, prevalence increased to $13.5 \%$ and $3.8 \%$, respectively (p-value $>0.05)$. These results are in contrast to a study by AlvaradoEsquivel et al. [11] from Mexico; they detected higher T. gondii seroprevalence $41.9 \%$ in young rabbits (age category: $0.3-1$ month) compared to older ones. Similar to Alvarado-Esquivel et al. [11], high T. gondii seropositivity of young domestic rabbits was confirmed by Uhliková and Hübner [4]; they also discussed the possibility of transplacental transmission of $T$. gondii in rabbits. The seroprevalence of T. gondii increased with the number of births, without statistical significant difference $(p$-value $=0.4141)$ however. This increasing trend of prevalence may be associated with post-natal infection through ingestion of food or water contaminated with T. gondii oocysts. The highest prevalence was found in hybrid line Y (30 \% for T. gondii) and X (14.4\% and 1.9\% for T. gondii and $N$. caninum, respectively), while $Z$ hybrids were negative for both T. gondii and N. caninum antibodies. The difference in $T$. gondii prevalence in breeds was statistically significant in some of breeds (test statistics $=12.64, \mathrm{df}=3$; $\mathrm{p}$-value $=0.005$ ), which is in contrast with the results of Alvarado-Esquivel et al. [11], who did not find statistical differences in breeds. The 
rabbits came from four provinces; the highest seroprevalence of T. gondii (40\%) and N. caninum (1.9\%) antibodies was found in Verona and Treviso provinces, respectively. In the case of $T$. gondii, the results were statistically different (test statistics $=42.08, \mathrm{df}=3$; p-value $<0.05$ ) between some of the provinces. Alvarado-Esquivel et al. [11] also found a statistically significant different $T$. gondii prevalence in rabbits from six municipalities in Mexico. This fact was explained by different climatic conditions. However, in the present study, there were no strong differences in climatic conditions of the six provinces, and the samples were collected on 13 farms. The highest T. gondii seroprevalence $(55 \%)$ was found in rabbits from one large farm in Verona province; the highest prevalence of $N$. caninum antibodies (5\%) was found on one small and two large farms in Treviso province. Nevertheless, there was no statistically significant difference (test statistics $=0.26$ resp. $0.20, \mathrm{df}=1 ; \mathrm{p}$-value $>0.05$ ) between either T. gondii and N. caninum seroprevalence and size of farms.

Two of 38 (5\%) rabbit does positive for T. gondii antibodies had been treated for respiratory or enteric disorders, which could be connected with symptoms of $T$. gondii infection. No signs of disease were recorded in the other does.

\section{CONCLUSION}

It is worth noting that rabbit does from Northern Italy are at risk of T. gondii and N. caninum infection which could be transmitted to their offspring that are mainly used for human consumption. That is why it is very important to implement good hygienic conditions on farms where the rabbits are bred. There was no data available about the presence of cats and dogs on the rabbit farms to study this risk factor. However, avoidance of the presence of cats and dogs on the farms is highly recommended to minimize the risk of contamination of the environment, food and water with T. gondii and N. caninum oocysts, respectively.

\section{Acknowledgements}

The study was funded by the Grant IZSME 05/10 RC C71J1000012000 from Italy, and Grant IMA 2014-FVHE17 from the Czech Republic.

\section{REFERENCES}

1. Dubey JP, Brown CA, Carpenter JL, Moore JJ. Fatal toxoplasmosis in domestic rabbits in the USA. Vet Parasitol. 1992; 44: 305-309.

2. Zanet S, Palese V, Trisciuoglio A, Cantón Alonso C, Ferroglio E. Neospora caninum: Detection in wild rabbits and investigation of co-infection with Toxoplasma gondii by PCR analysis. Vet Parasitol. 2013; 197: 682-684.

3. Dubey JP. Toxoplasmosis of animals and humans, second edition. CRC Press, 2010, Taylor and Francis Group, Boca Raton, Florida ISBN 978-1-4200-9236-3, p. 313.

4. Uhliková M, Hübner J. Congenital transmission of toxoplasmosis in domestic rabbits. Folia Parasitol. 1973; 20: 285-291.

5. Lonardi C, Grilli G, Ferrazzi V, Dal Cin M, Rigolin D, Piccirillo A. Serological survey of Encephalitozoon cuniculi infection in commercially reared rabbit does in Northern Italy. Res Vet Sci. 2013; 94: 295-298.

6. Sedlak K, Literak I, Faldyna M, Toman M, Benak J. Fatal toxoplasmosis in brown hares (Lepus europaeus): possible reason of their high susceptibility to the infection. Vet Parasitol. 2000; 93: 13-28.

7. StatSoft, Inc. (2013). STATISTICA (data analysis software system), version 12. www.statsoft.com.

8. Zhou Y, Zhang H, Cao J, Gong H, Zhou J. Isolation and genotyping of Toxoplasma gondii from domestic rabbits in China to reveal the prevalence of type III strains. Vet Parasitol. 2013; 193: 270-276.

9. Neumayerova H, Jurankova J, Jeklova E, Kudlackova H, Faldyna M, Kovarcik K et al.. Seroprevalence of Toxoplasma gondii and Encephalitozoon cuniculi in rabbits from different fading systems. Vet Parasitol. 2014; 204: 184-190.

10. Ashmawy KI, Abuakkada SS, Awad AM. Seroprevalence of antibodies to Encephalitozoon cuniculi and Toxoplasma gondii in farmed domestic rabbits in Egypt. Zoon Publ Health. 2010; 58: 357-364.

11. Alvarado-Esquivel C, Alvarado-Esquivel D, Villena I, Dubey JP. Seroprevalence of Toxoplasma gondii infection in domestic rabbits in Durango State, Mexico. Prev Vet Med. 2013; 111: 325-328. 